\title{
Schilddrüse in speziellen Situationen
}

\author{
Peter Mikosch
}

Online publiziert: 19. Oktober 2020

(C) Springer-Verlag GmbH Austria, ein Teil von Springer Nature 2020

Die Indikationsstellung zur Schilddrüsenoperation und vor allem in welchem Ausmaß die Schilddrüse reseziert werden soll, ist Thema anhaltender Diskussionen. In deren Beitrag „Moderne Schilddrüsenchirurgie - das endokrin-chirurgische Verständnis des Operateurs und seine Verantwortung für das Resektionsausmaß und Komplikationsrate" stellen Hermann et al. in differenzierter Weise das chirurgische Vorgehen bei den einzelnen Indiktionen zu einer Schilddrüsenoperation dar. Die notwendige Radikalität einer Schilddrüsenoperation wird den möglichen Komplikationen gegenübergestellt. Die Autoren fordern eine enge Zusammenarbeit zwischen den Diagnostikern und den Chirurgen sowie frühzeitige Einbindung des Chirurgen. Um die differenzierten Entscheidungsfindungen betreffend Resektionsausmaß ausreichend gut treffen $\mathrm{zu}$ können, fordern die Autoren des Weiteren ein ausreichendes Verständnis und Eigenerfahrung betreffend Sonographie und Labordiagnostik vom Chirurgen.

Jod spielt eine essentielle Rolle bei der Schilddrüsenhormonsynthese. Jodmangelzustände haben in der Vergangenheit in vielen Regionen der Welt Jodmangelstrumen hervorgerufen. Durch die Einführung der Zugabe von Jod zu Lebensmitteln, so z. B. in Österreich durch Jodierung des Speisesalzes, konnten diese Jodmangelzustände und die Inzidenz der Jodmangelstruma deutlich gesenkt werden. Demgegenüber können jedoch zu hohe Jodbelastungen zu einer Häufung von Immunthyreopathien, Dekompensation von autonomen Adenomen führen. Mikosch et al.

Prim. Univ.-Prof. Dr. P. Mikosch ( $₫)$

Teaching Unit, Medizinische Universität Wien/Medical University Vienna, Wien, Österreich

Innere Medizin 2, Landesklinikum Mistelbach-Gänserndorf, Liechtensteinstraße 67, 2130 Mistelbach, Österreich peter.mikosch@meduniwien.ac.at beleuchten in deren Artikel über „Kuranwendungen mit jodhaltigen Thermalwasser aus thyreologischer Sicht" die bislang in der Literatur wenig beachtete Konstellation einer gezielten Jodzufuhr im Rahmen von Kuranwendungen mit jodhaltigem Thermalwasser. Neben positiven Effekten einer Kuranwendung mit jodhaltigen Thermalwassern werden mögliche negative Auswirkungen auf die Schilddrüse und deren Funktion dargestellt. Für den täglichen klinischen Einsatz bei der Abwägung positiver versus negativer Auswirkungen auf die Schilddrüse werden Kurorte mit jodhaltigen Thermalwasser aufgelistet und ein Workflow vorgestellt, um Patienten mit eventueller Gefährdung betreffend einer Anwendung mit jodhaltigen Thermalwassern noch vor Kurantritt zu erfassen.

Die Hemiagenesie der Schilddrüse stellt eine seltene Anlageanomalie der Schilddrüse dar. Die meisten in der Literatur beschriebenen Fälle wurden durch weitere Erkrankungen im vorhandenen Schilddrüsenlappen festgestellt. Anhand eines Fallberichts, bei dem der Patient mit Hemiagenesie der Schilddrüse keine weitere Schilddrüsenerkrankung aufwies, stellten Mikosch et al. in deren Manuskript die Frage, welche Auswirkungen eine Hemiagenesie der Schilddrüse für sich alleine haben kann.

Eine weitere spezielle Situation stellt die Schilddrüse im höheren Alter dar. Im Beitrag „Schilddrüse im Alter - Physiologie und Pathologie" von Tugendsam und Kurtaran geben die Autoren einen Überblick von Schilddrüsenerkrankungen im Alter. Die Frage nach altersspezifischen TSH-Werten wird beleuchtet und darauf aufbauend ein differenziertes therapeutisches Vorgehen bei der Behandlung von Schilddrüsenfehlfunktionen bei alten Menschen präsentiert. Die altersassoziierten Veränderungen des Schilddrüsenstoffwechsels werden des Weiteren mit im höheren Alter regelmäßig vorkommenden Komorbiditäten 


\section{editorial}

verknüpft und ein auf den alten Menschen adaptiertes Therapiemanagment dargestellt.

Als Editor dieses Schilddrüsen-Themenheftes verbleibe ich in der Hoffnung, dem Leser interessante und neue Einblicke in die dargestellten speziellen Themenbereiche der Schilddrüse übermitteln zu können.
Interessenkonflikt P. Mikosch gibt an, dass kein Interessenkonflikt besteht.

Hinweis des Verlags Der Verlag bleibt in Hinblick auf geografische Zuordnungen und Gebietsbezeichnungen in veröffentlichten Karten und Institutsadressen neutral. 